\title{
Seguimiento de paciente con enfermedad de Chagas y trasplante de corazón mediante las PCR S35-S36 y TcH2AF-R
}

\author{
Paula Ximena Pavía', Nubia Lucía Roa², Ana María Uribe³ ${ }^{3}$ Concepción Judith Puerta \\ 1 Laboratorio de Parasitología Molecular, Departamento de Microbiología, Facultad de Ciencias, Pontificia \\ Universidad Javeriana, Bogotá, D.C., Colombia \\ 2 Clínica de Falla y Trasplante Cardiaco, Departamento de Cardiología, Hospital Universitario San Ignacio, \\ Pontificia Universidad Javeriana, Bogotá, D.C., Colombia \\ 3 Laboratorio de Patología, Hospital Universitario San Ignacio, Pontificia Universidad, Javeriana, Bogotá, D.C., \\ Colombia
}

Introducción. La cardiomiopatía es la forma clínica más común de la enfermedad de Chagas en Colombia, siendo el trasplante una opción para su tratamiento. Debido al riesgo de reactivación de la infección posterior al trasplante, es prioritario vigilar el comportamiento del parásito.

Objetivo. Presentar el caso de un paciente con cardiopatía chagásica dilatada y falla cardiaca, a quien se le practicó trasplante de corazón y se le hizo seguimiento mediante PCR, análisis histopatológicos y ecocardiográficos.

Materiales y métodos. Se tomaron muestras de sangre antes de la intervención y después de ella y de biopsias endomiocárdicas posteriores al trasplante. El ADN extraído fue amplificado con los iniciadores TcH2AF-R y S35-S36. La parasitemia se examinó mediante la técnica de microhematocrito. Se practicaron estudios histopatológicos para determinar la presencia del parásito o el rechazo del trasplante y, ecocardiográficos, para evaluar la función cardiaca.

Resultados. De las muestras de sangre tomadas a los 83 y 48 días previos al trasplante, la última fue positiva por la PCR S35-S36. Hasta el segundo mes después del trasplante, ambas PCR fueron negativas. Al tercer mes después del trasplante, ambas PCR fueron positivas, por lo cual se inició tratamiento con nifurtimox. Tras 35 días de haberse iniciado el tratamiento, ambas pruebas presentaron resultados negativos, al igual que las tomadas a los 0, 3, 10 y 12 meses posteriores. Los resultados de la histopatología, del microhematocrito y de las PCR de las biopsias, fueron negativos en todas las fechas.

Conclusiones. Las PCR permitieron sospechar la reactivación de la infección en el paciente, se le administró el tratamiento y posterioremente las pruebas se tornaron negativas. La evolución clínica del paciente ha sido favorable.

Palabras clave: Trypanosoma cruzi, enfermedad de Chagas, trasplante, reacción en cadena de la polimerasa (PCR), ecocardiografía, cardiomiopatía dilatada.

Using S35-S36 and TcH2AF-R primer-based PCR tests to follow-up a Chagas' disease patient who had undergone a heart transplant

Introduction. Cardiomyopathy is the most common clinical form of Chagas' disease in Colombia, and one treatment option is a heart transplant. Tracking the behavior of the Chagas' parasite, Trypanosoma cruzi, is a priority due to the risk of post-transplant reactivation of the infection.

Objective. A case is presented of a patient who had suffered from dilated chagasic cardiopathy and cardiac failure, and had subsequently undergone heart transplant. The case was monitored by PCR, histopathological and echocardiographic examinations.

Materials and methods. Blood samples were drawn before and after the transplant, and post-transplant endomyocardial biopsies were taken. The extracted DNA was amplified with the TcH2AF-R and S35S36 primers. Parasitemia was examined by the microhematocrit test. In addition, histopathological studies determined the parasite presence and transplant rejection status. Echocardiograms were administered to evaluate cardiac function.

Results. Of the blood samples taken 83 and 48 days pre-transplant, the latter was positive by the S35-S36 PCR test. PCR tests in blood with both primers were negative up to the second month posttransplant. However, both PCR tests were positive by the third month post-transplant. Thereupon, the patient was treated with nifurtimox. Both tests presented negative results in blood 35 days after treatment was started and remained negative thereafter at 0, 3, 10 and 12 months post-treatment. The pathology, microhematocrit, and PCR test results from biopsies were negative on all the specified dates. 
Conclusions. PCR tests were used as indicators of a reactivation of trypanosomid infection in the patient. After treatment administration, PCR tests became negative. The patient's clinical evolution was favorable.

Key words: Trypanosoma cruzi, Chagas disease, transplantation, polymerase chain reaction, echocardiography; cardiomiopathy, dilated.

La enfermedad de Chagas constituye uno de los problemas de salud pública más importante en Centroamérica y Suramérica. Alrededor de 8 millones de personas se encuentran infectadas, se presentan, aproximadamente, 41.200 nuevos casos por año y 108 millones de individuos se encuentran en riesgo de adquirir la enfermedad (1). En Colombia, actualmente se estima que existen, aproximadamente, 436.000 infectados, de los cuales, aproximadamente, 131.474 cursan con cardiopatía chagásica (2).

Durante el curso de la infección por Trypanosoma cruzi, 10 a $30 \%$ de las personas con serología positiva pueden presentar cambios electrocardiográficos 10 a 20 años después de la infección y $10 \%$ de los individuos pueden morir por la evolución a cardiopatía chagásica crónica $(3,4)$. En Colombia, la forma más común de la enfermedad es la cardiopatía, caracterizada por arritmias leves a graves, miocarditis, cardiomiopatía dilatada, falla cardiaca y muerte súbita (5). La fisiopatología de la cardiomiopatía dilatada es compleja y parcialmente comprendida. Los pacientes con esta afección pueden progresar a insuficiencia cardiaca resistente a un manejo médico óptimo, correspondiente al estadio $D$ de la falla cardiaca (6), y requerir de trasplante cardiaco (7). Después del trasplante, los pacientes corren gran riesgo de presentar reactivación de la infección de manera temprana, debido a las condiciones de inmunosupresión a que se ven sometidos para tal procedimiento (8-10).

El seguimiento del paciente con enfermedad de Chagas y trasplante para identificar reactivación de la infección, se hace principalmente mediante análisis parasitológicos directos, como microhematocrito, hemocultivo y xenodiagnóstico, estudio histopatológico y pruebas de reacción en cadena de la

\footnotetext{
Correspondencia:

Concepción Judith Puerta, Laboratorio de Parasitología Molecular, Departamento de Microbiología, Facultad de Ciencias, Pontificia Universidad Javeriana, Bogotá, D.C. Colombia

Teléfono:(+571) 320 8320, extensión 4024; fax (571) 3208320 , extensión 4021

cpuerta@javeriana.edu.co

Recibido: 09/08/10; aceptado:11/02/11
}

polimerasa (PCR) en sangre o tejidos, siendo el xenodiagnóstico y el hemocultivo los menos utilizados por su menor sensibilidad y tiempo requerido no menor de 30 días para obtener el resultado (11). El estudio histopatológico es uno de los métodos más utilizados para detectar T. cruzi en lesiones del tejido cardiaco y otros. Sin embargo, no siempre se observan formas intracelulares del parásito (12). La PCR ha sido utilizada para detectar T. cruzien tejidos de pacientes con enfermedad de Chagas, dadas sus ventajas en términos de sensibilidad y especificidad (9,12-15).

En este trabajo se presenta el seguimiento de un paciente con cardiomiopatía chagásica sometido a trasplante cardiaco, en quien se siguió el comportamiento del parásito mediante las PCR TcH2AF-R y S35-S36, específicas para T. cruzi (16-20).

\section{Presentación del caso}

Se trata de una mujer de 55 años de edad natural de Soatá (Boyacá) con diagnóstico de enfermedad de Chagas confirmado por pruebas serológicas y cardiomiopatía crónica dilatada, que consultó al servicio de urgencias del Hospital Universitario San Ignacio de Bogotá, en noviembre de 2007, por síndrome de falla cardiaca aguda con compromiso grave de la fracción de eyección (28\%) de tres años de evolución.

Se procedió a practicar trasplante cardiaco en mayo del 2008. Para dicho procedimiento, se hizo inducción con ciclosporina oral, daclizumab y metilprednisolona. En el periodo posoperatorio la paciente recibió como inmunomoduladores prednisolona, ciclosporina y micofenolato de mofetilo, y por protocolo, recibió profilaxis para infecciones por citomegalovirus, Pneumocystis jiroveciiy Aspergillus fumigatus, con valganciclovir, trimetoprim-sulfametoxazol e itraconazol por los primeros tres meses.

\section{Materiales y métodos \\ Muestras analizadas}

Se tomaron dos muestras de sangre antes de la intervención, a los 83 y 48 días antes del trasplante. Las muestras de sangre y de la biopsia 
de endomiocardio se tomaron 17 y 25 días después del trasplante, quincenalmente durante los siguientes dos meses, mensualmente hasta los seis meses, y a los 9, 16 y 18 meses. Ambos tipos de muestras fueron analizadas mediante las PCR TcH2AF-R y S35-S36. Las muestras de sangre fueron analizadas también mediante la técnica de microhematocrito para la búsqueda del parásito.

\section{Análisis histopatológico}

El análisis histopatológico de las biopsias endomiocárdicas se hizo en el Laboratorio de Patología del Hospital Universitario San Ignacio, mediante las técnicas de hematoxilina y eosina convencional y coloración de tricrómico. El rechazo en la histopatología se determinó de acuerdo con la clasificación de la International Society for Heart and Lung Transplantation (21).

\section{Análisis por reacción en cadena de la polimerasa}

EI ADN de $1 \mathrm{ml}$ de sangre fue extraído por el método de triple fenol-cloroformo-alcohol isoamílico, seguido de la precipitación de ADN con etanol absoluto y, aproximadamente, el de $10 \mu \mathrm{m}$ de las biopsias, por el método de salting out (22). El ADN obtenido, concentrado y diluido 1/5, 1/10, 1/20 y 1/40, fue amplificado con los iniciadores TcH2AF (5'-GAGAGTGATCGTGGGAGAGCA-3') y TcH2AR (5'-AGTGGCAGACTTTGGGGTC-3'), los cuales amplifican una banda de $234 \mathrm{pb}$ del elemento SIRE ubicado en la región 3' no codificante de la unidad de $1,2 \mathrm{~kb}$ del gen histona $H 2 A(16)$, y los iniciadores $S 35$ (5'-AAATAATGTACGGGTGGAGATGCATGA-3') y S36 (5'-GGGTTCGATTGGGGTTGGTGT-3'), los cuales amplifican un fragmento de $330 \mathrm{pb}$ correspondiente a la región variable de los minicírculos de T. cruzi (20).

Las reacciones de amplificación se llevaron a cabo de acuerdo con lo descrito por Pavía et al. (2007), utilizando los siguientes controles: control negativo, en el que se adicionó agua en lugar de ADN; control positivo, ADN de T. cruzi de formas de cultivo; control humano, ADN de un individuo sano, y control de la extracción del ADN, en el cual se utilizó agua en lugar de sangre durante el procedimiento de extracción (17). Los productos de amplificación se analizaron mediante electroforesis en geles de agarosa al 1,5 \% teñidos con bromuro de etidio. Para descartar la presencia de inhibidores de la PCR en las muestras negativas, a éstas se les adicionó ADN del parásito, y luego se hizo la respectiva amplificación.

\section{Resultados}

\section{Seguimiento de la reactivación de la infección por $T$. cruzi después del trasplante}

Antes del trasplante, se le tomaron al paciente dos muestras de sangre en fechas diferentes, y solamente la última muestra (48 días antes del trasplante) fue positiva con los iniciadores S35-S36. Hasta el segundo mes después del trasplante, las PCR en sangre con ambos iniciadores presentaron resultados negativos. Al tercer mes posterior al trasplante, ambas PCR, TcH2AF-R y S35-S36, fueron positivas en sangre, observándose los productos de amplificación específicos de 234 y $330 \mathrm{pb}$, respectivamente (figura 1).

Con base en estos resultados, se formuló tratamiento con nifurtimox (Lampit @), 8 a $10 \mathrm{mg} / \mathrm{kg}$, durante 60 días, y tras 18 días de haberlo iniciado,

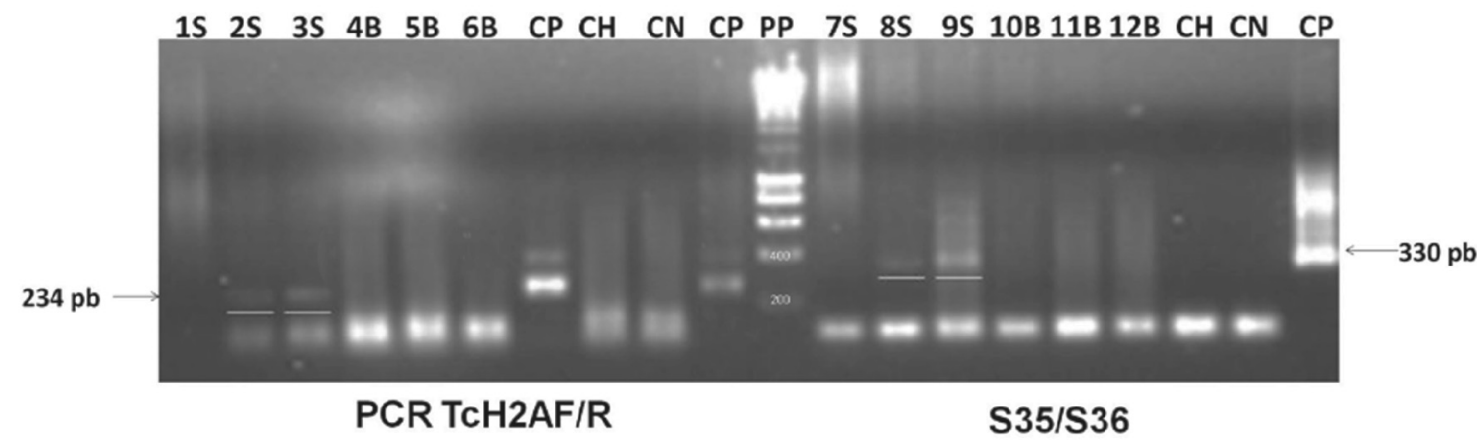

Figura 1. Resultado de las PCR TcH2A-R y S35-S36 en las muestras de sangre (S) y biopsia (B) a los 3 meses después del trasplante. Electroforesis horizontal en gel de agarosa al 1,5\% coloreado con bromuro de etidio que contiene: $20 \mu \mathrm{l}$ del producto de amplificación de S sin dilución (bandas 1 y 7), S con dilución 1 en 5 (bandas 2 y 8), S con dilución 1 en 10 (bandas 3 y 9), B sin dilución (bandas 4 y 10), B con dilución 1 en 5 (bandas 5 y 11), B con dilución 1 en 10 (bandas 6 y 12). Control positivo: ADN de la cepa MHOM/CO/86/MR de T. cruzi (CP); control humano: ADN de un individuo sano (CH); control negativo: agua destilada (CN); patrón de peso molecular (PP): Hyper Ladder II (Bioline®, Londres, Reino Unido). 
ambas PCR continuaron positivas en sangre, con resultados negativos 27 días más tarde. Se continuó el seguimiento del paciente, y se observó al finalizar el tratamiento y a los 3, 10 y 12 meses después de finalizado (270, 480 y 540 días después del trasplante) que ambas PCR permanecieron negativas en sangre (figura 2). Los resultados de microhematocrito, histopatología y PCR de la biopsia endomiocárdica, fueron negativos para $T$. cruzi en todas las fechas.

\section{Hallazgos histopatológicos}

Las biopsias mostraron presencia de infiltrado inflamatorio mononuclear, difuso, en los días 90 y 120 después del trasplante, lo cual se clasifica como rechazo celular agudo leve (1R).

En el estudio histopatológico, a los 150 y 180 días después del trasplante, se observó aumento del infiltrado inflamatorio, con un foco agresivo que lesionaba la fibra miocárdica, lo que se interpretó como rechazo celular agudo leve con mayor inflamación que la vista en las biopsias anteriores. Con la coloración de tricrómico se identificó fibrosis importante, la cual no se esperaba ver de acuerdo con el tiempo de evolución del trasplante. Las biopsias de las siguientes fechas (270 y 360 días) fueron calificadas como $1 \mathrm{R}$ y $0 \mathrm{R}$, respectivamente (cuadro 1). No se identificó el microorganismo en ninguna de las biopsias analizadas.

\section{Hallazgos ecocardiográficos}

En el seguimiento ecocardiográfico no se encontró alteración hasta el estudio realizado a los 75 días después del trasplante, cuando se presentaron por primera vez insuficiencia mitral leve, dilatación del ventrículo derecho y ligera disminución de la fracción de eyección del ventrículo izquierdo (50 $\%)$. En los estudios practicados posteriormente se encontró aumento de la insuficiencia mitral hasta GIII/IV y fracción de eyección del ventrículo izquierdo adecuada. En el estudio practicado a los 180 días, se encontró disminución de la fracción de eyección a $45 \%$, con persistencia de la insuficiencia mitral GIII/IV. En los controles a los 270 y 360 días, se encontró deterioro en la fracción de eyección (cuadro 1).

\section{Alteraciones clínicas después del trasplante}

El paciente tuvo una evolución clínica adecuada hasta los 90 días después del trasplante, sin signos de falla cardiaca, miocarditis, fiebre ni nódulos subcutáneos que pudieran asociarse con reactivación de la infección o rechazo agudo. A los 90 días se encontró leve congestión sistémica, dada por hepatomegalia e ingurgitación yugular, sin disnea. En las siguientes consultas no se encontró ningún signo ni síntoma que sugiriera falla cardiaca o reactivación de la infección por $T$. cruzi.

\section{Discusión}

A pesar de la controversia previamente existente sobre someter a trasplante cardiaco a los pacientes con cardiomiopatía de origen chagásico, se ha visto que estos pacientes presentan menor frecuencia de rechazo $(23,24)$ y supervivencia similar $(10,14,24,25)$, e incluso superior según algunas series, a la de los pacientes no chagásicos (7). Además, las complicaciones del trasplante, como neoplasias o infecciones, no parecen ser más frecuentes o graves en ellos $(23,24,26,27)$. Más aún, los estudios recientes de Bertolino et al. informan que $30 \%$ de los enfermos con enfermedad de Chagas en lista de espera de trasplante de corazón, tienen un desenlace fatal, frente al 16 $\%$ de los que no presentan esta enfermedad en iguales condiciones (28). Por lo tanto, actualmente el trasplante es considerado una de las opciones

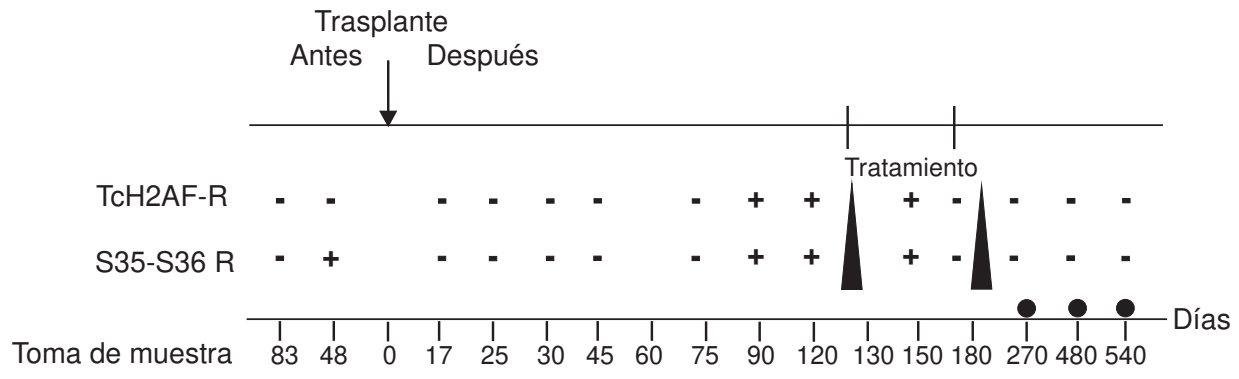

Figura 2. Esquema de muestreo y resultados de las PCR TcH2AF-R y S35-S36 antes y después del trasplante, y después del tratamiento. Los días corresponden al momento en que se obtuvieron las muestras de sangre en relación con la fecha del trasplante, éste último indicado mediante una flecha. Los triángulos indican el periodo del tratamiento y los días en los que se tomaron muestras después del tratamiento, correspondientes a 270, 480 y 540 días después del trasplante, respectivamente, se señalan con un círculo. 
Cuadro 1. Seguimiento de la evolución del trasplante y de la función cardiaca del paciente.

\begin{tabular}{|c|c|c|}
\hline \multirow[b]{2}{*}{$\begin{array}{l}\text { Días después } \\
\text { del trasplante }\end{array}$} & \multicolumn{2}{|l|}{ Hallazgos clínicos } \\
\hline & Biopsia endomiocárdica & Ecocardiograma \\
\hline 7 & $\begin{array}{l}\text { Rechazo celular leve } 1 \mathrm{R} \\
\text { Lesión isquémica temprana, sospecha de rechazo } \\
\text { humoral; con soporte inotrópico }\end{array}$ & Fracción de eyección, 50 \% \\
\hline 17 & $\begin{array}{l}\text { Rechazo celular leve 1R } \\
\text { Insuficiencia mitral }\end{array}$ & Fracción de eyección, 45 \% \\
\hline 25 & Rechazo celular leve $1 \mathrm{R}$ & Fracción de eyección, 60 \% \\
\hline 30 & Rechazo celular leve OR & Fracción de eyección, 50 \% \\
\hline 45 & Rechazo celular leve $0 \mathrm{R}$ & Fracción de eyección, 55 \% \\
\hline 60 & Rechazo celular leve $0 \mathrm{R}$ & Fracción de eyección, 50 \% \\
\hline 75 & $\begin{array}{l}\text { Rechazo celular leve } 0 \mathrm{R} \\
\text { Sin evidencia de proceso inflamatorio }\end{array}$ & $\begin{array}{l}\text { Fracción de eyección, } 50 \text { \% } \\
\text { Insuficiencia mitral }\end{array}$ \\
\hline 90 & $\begin{array}{l}\text { Rechazo celular leve } 1 \mathrm{R} \\
\text { Infiltrado inflamatorio mononuclear intersticial difuso } \\
\text { perivascular, sin evidencia de lesión de la fibra miocárdica }\end{array}$ & Fracción de eyección, 55 \% \\
\hline 120 & $\begin{array}{l}\text { Rechazo celular leve } 1 \mathrm{R} \\
\text { Infiltrado inflamatorio mononuclear intersticial difuso } \\
\text { perivascular, sin evidencia de lesión de la fibra miocárdica }\end{array}$ & $\begin{array}{l}\text { Fracción de eyección, } 55 \text { \% } \\
\text { Dilatación ventricular izquierda }\end{array}$ \\
\hline 150 & $\begin{array}{l}\text { Rechazo celular leve } 1 \mathrm{R} \\
\text { Fibrosis con atrapamiento de fibras miocárdicas, } \\
\text { infiltrado inflamatorio mononuclear intersticial difuso } \\
\text { perivascular, con lesiones de la fibra miocárdica. }\end{array}$ & Fracción de eyección, 50 \% \\
\hline 180 & $\begin{array}{l}\text { Rechazo celular leve } 1 \mathrm{R} \\
\text { Foco agresivo perivascular con infiltrado inflamatorio } \\
\text { mononuclear intersticial difuso }\end{array}$ & Fracción de eyección, 45 \% \\
\hline 270 & $\begin{array}{l}\text { Rechazo celular leve } 1 \mathrm{R} \\
\text { Infiltrado inflamatorio mononuclear intersticial difuso } \\
\text { perivascular, sin evidencia de lesión de la fibra miocárdica, } \\
\text { tejido fibroso laxo con atrapamiento de fibras miocárdicas } \\
\text { correspondientes a sitios de biopsia previa }\end{array}$ & $\begin{array}{l}\text { Fracción de eyección, } 23 \% \\
\text { Suspensión por } 30 \text { días de prednisolona } \\
\text { por parte de la paciente, dilatación de } \\
\text { ventrículo izquierdo }\end{array}$ \\
\hline 360 & $\begin{array}{l}\text { Rechazo celular leve } \mathrm{OR} \\
\text { Fibras musculares atrapadas por tejido fibroso }\end{array}$ & Fracción de eyección, 25 \% \\
\hline
\end{tabular}

de tratamiento indicada para los pacientes con cardiomiopatía chagásica crónica (11,27-29).

La reactivación de la infección causada por $T$. cruzi es una amenaza para la supervivencia del injerto y del paciente; ésta se puede presentar con una frecuencia de 8 a $90 \%(11,26)$, diagnosticándose con mayor frecuencia en el primer año después del trasplante, especialmente en los primeros tres meses cuando el tratamiento de inmunosupresión es más intenso (11).

En el seguimiento de la reactivación de la infección por $T$. cruzi en pacientes con trasplante, se ha observado que la PCR tiene mayor sensibilidad que los métodos convencionales directos $y$ ha permitido detectar el parásito de forma temprana, antes de que aparezcan los síntomas asociados a la reactivación de la enfermedad (14,30). De acuerdo con lo anterior, los resultados del microhematocrito en este estudio fueron negativos en todas las fechas. Por el contrario, ambas PCR permitieron detectar el parásito a los 90 días después del trasplante. Más aún, la PCR TcH2AF-R -de menor sensibilidad que la prueba S35-S36- cambió de negativa en las muestras antes del trasplante y después de él hasta los dos meses, a positiva en la muestra tres meses después del trasplante, resultado que podría explicarse por un aumento de la parasitemia.

Por otra parte, la falta de identificación del parásito en las biopsias endomiocárdicas podría atribuirse a que éste estuviese iniciando el proceso de invasión al tejido cardíaco, proceso que se lleva a cabo a través del epicardio (12).

En resumen, en este estudio se consideró la reactivación de la infección a los tres meses después del trasplante debido al viraje en los resultados de las PCR en sangre con persistencia del resultado positivo hasta los 120 días, a pesar de la ausencia de síntomas asociados a la infección. El grupo tratante decidió iniciar tratamiento antiparasitario, tras el cual se observó que las PCR se tornaron negativas y así permanecieron. 
El paciente clínicamente ha permanecido en adecuada clase funcional, sin signos de falla cardiaca, ni de reactivación de la enfermedad de Chagas, a pesar de los hallazgos ecocardiográficos que muestran compromiso de la fracción de eyección.

Este caso representa el primer reporte en Colombia de seguimiento de trasplante cardíaco en pacientes con enfermedad de Chagas mediante PCR. Dada la baja sensibilidad de las otras pruebas diagnósticas, la PCR será de mucha utilidad para el diagnóstico temprano de reactivación de la infección en pacientes inmunosuprimidos, en quienes el tiempo para iniciar el tratamiento es determinante para el control de la infección.

\section{Agradecimientos}

Los autores expresan su agradecimiento al paciente y a las Unidades de Hemodinamia Clínica y Cardiología del Hospital Universitario San Ignacio.

\section{Conflicto de interés}

Los autores del presente artículo declaramos no tener conflictos de intereses de orden académico, institucional u operaciotivo, durante esta la investigación.

\section{Financiación}

El estudio fue desarrollado como parte del proyecto 00001034 financiado por la Pontificia Universidad Javeriana.

\section{Referencias}

1. World Health Organization. Report of the Scientific Working Group on Chagas Disease. Buenos Aires, Argentina, 17-20 April 2005. Update. Geneva: World Health Organization; July 2007. Fecha de consulta: abril de 2010. Disponible en: http:// www.who.int/tdrold/diseases/chagas/swg_chagas.pdf.

2. Organización Panamericana de la Salud. Estimación cuantitativa de la enfermedad de Chagas en las Américas. Washington, D.C.: OPS; 2006.

3. World Health Organization. Control of Chagas disease. Report of a WHO Expert Committee. Technical Report Series. Geneva: World Health Organization; 2002.

4. Bilate AM, Cunha-Neto E. Chagas disease cardiomyopathy: Current concepts of an old disease. Rev Inst Med Trop Sao Paulo. 2008;50:67-74

5. Moncayo A. Chagas disease: Current epidemiological trends after the interruption of vectorial and transfusional transmission in the Southern Cone countries. Mem Inst Oswaldo Cruz. 2003;98:577-91.

6. Gómez EA, Senior JM, Vélez S, Navarrete S, Sánchez D, Roa NL, et al. Guías colombianas sobre la evaluación y el manejo de la falla cardíaca crónica del adulto. Revista Colombiana de Cardiología. 2007;14:13-50.
7. Bocchi EA, Fiorelli A. The paradox of survival results after heart transplantation for cardiomyopathy caused by Trypanosoma cruzi. First Guidelines Group for Heart Transplantation of the Brazilian Society of Cardiology. Ann Thorac Surg. 2001;71:1833-8.

8. Phan BA, Laflamme MA, Otero AS, Limaye AP, Buckner FS, Levy WC. Confirmation of Chagas' cardiomyopathy following heart transplantation. Heart Vessels. 2006;21:325-7.

9. Benvenuti LA, Roggério A, Sambiase NV, Fiorelli A, Higuchi ML. Polymerase chain reaction in endomyocardial biopsies for monitoring reactivation of Chagas' disease in heart transplantation: A case report and review of the literature. Cardiovasc Pathol. 2005;14:265-8.

10. Almeida DR, Carvalho AC, Branco JN, Pereira AP, Correa L, Vianna PV, et al. Chagas' disease reactivation after heart transplantation: Efficacy of allopurinol treatment. J Heart Lung Transplant. 1996;15:988-92.

11. Bacal F, Silva CP, Pires PV, Mangini S, Fiorelli Al, Stolf NG, et al. Transplantation for Chagas' disease: An overview of immunosuppression and reactivation in the last two decades. Clin Transplant. 2010;24:E29-34.

12. Schijman AG, Vigliano CA, Viotti RJ, Burgos JM, Brandariz S, Lococo BE, et al. Trypanosoma cruzi DNA in cardiac lesions of Argentinean patients with endstage chronic chagas heart disease. Am J Trop Med Hyg. 2004;70:210-20.

13. Benvenuti LA, Roggério A, Freitas HF, Mansur AJ, Fiorelli A, Higuchi ML. Chronic American trypanosomiasis: Parasite persistence in endomyocardial biopsies is associated with high-grade myocarditis. Ann Trop Med Parasitol. 2008;102:481-7.

14. Diez M, Favaloro L, Bertolotti A, Burgos JM, Vigliano C, Lastra MP, et al. Usefulness of PCR strategies for early diagnosis of Chagas' disease reactivation and tratament follow-up in heart transplantation. Am J Transplant. 2007;7:1633-40.

15. Elias FE, Vigliano CA, Laguens RP, Levin MJ, Berek C. Analysis of the presence of Trypanosoma cruzi in the heart tissue of three patients with chronic Chagas' heart disease. Am J Trop Med Hyg. 2003;68:242-7.

16. Pavía P, Cuervo C, Montilla M, Nicholls RS, Puerta C. Diseño y estandarización de una PCR para la detección específica de Trypanosoma cruzi. Infectio. 2003;7:12936.

17. Pavia P, Vallejo GA, Montilla M, Nicholls RS, Puerta CJ. Detection of Trypanosoma cruzi and Trypanosma rangeli infection in triatomine vectors by amplification histone $\mathrm{H} 2 \mathrm{~A}$ and sno-RNA-CL1 genes. Rev Inst Med Trop Sao Paulo. 2007;49:23-30.

18. Gil J, Pavía P, Montilla M, Florez AC, Quintero C, Mercado M, et al. Comparison of a PCR test based on the histone H2A/SIRE genes with classical serological tests for the diagnosis of chronic Chagas disease in Colombian patients. Biomedica. 2007;27(Suppl.1):83-91.

19. Barrera YK, Guevara JM, Pavía PX, Montilla M, Nicholls RS, Parra E, et al. Evaluation of TcH2AF-R and S35-S36 primers in PCR tests for the detection of Trypanosoma cruzi in mouse cardiac tissue. Biomédica. 2008;28:616-26. 
20. Sturm NR, Degrave W, Morel C, Simpson L. Sensitive detection and schizodeme classification of Trypanosoma cruzi cells by amplification of kinetoplast minicircle DNA sequences: Use in diagnosis of Chagas disease. Mol Biochem Parasitol. 1989;33:205-14.

21. Stewart S, Winters GL, Fishbein MC, Tazelaar HD, Kobashigawa $\mathrm{J}$, Abrams $\mathrm{J}$, et al. Revision of the 1990 Working Formulation for the Standardization of Nomenclature in the Diagnosis of Heart Rejection. J Heart Lung Transplant. 2005;24:1710-20.

22. Aljanabi SM, Martínez I. Universal and rapid salt-extraction of high quality genomic DNA for PCR-based techniques. Nucleic Acids Res. 1997;25:4692-3.

23. de Carvalho VB, Sousa EF, Vila JH, da Silva JP, Caiado MR, Araujo SR, et al. Heart transplantation in Chagas' disease. 10 years after the initial experience. Circulation. 1996;94:1815-7.

24. Bestetti RB, Theodoropoulos TA. A systematic review of studies on heart transplantation for patients with end-stage Chagas' heart disease. J Card Fail. 2009;15:249-55.

25. Urinovsky F, Salomone O, Córdoba R, Zazu A, Columbres A, Zlocowsky J, et al. Mortalidad de los pacientes con cardiopatía chagásica y trasplante cardiaco. Experiencia inicial. Revista Argentina de Cardiología. 2003;71:325-31.

26. Bestetti RB, Souza TR, Lima MF, Theodoropoulos TA, Cordeiro JA, Burdmann EA. Effects of a mycophenolate mofetil-based immunosuppressive regimen in Chagas' heart transplant recipients. Transplantation. 2007;84:441-2.

27. Biolo A, Ribeiro AL, Clausell N. Chagas cardiomyopathy -where do we stand after a hundred years? Prog Cardiovasc Dis. 2010;52:300-16.

28. Bertolino ND, Villafanha DF, Cardinalli-Neto A, Cordeiro JA, Arcanjo MJ, Theodoropoulos TA, et al. Prognostic impact of Chagas' disease in patients awaiting heart transplantation. J Heart Lung Transplant. 2010;29:449-53.

29. Godoy HL, Guerra CM, Viegas RF, Dinis RZ, Branco JN, Neto VA, et al. Infections in heart transplant recipients in Brazil: The challenge of Chagas' disease. J Heart Lung Transplant. 2010;29:286-90.

30. Maldonado C, Albano S, Vettorazzi L, Salomone O, Zlocowski JC, Abiega C, et al. Using polymerase chain reaction in early diagnosis of re-activated Trypanosoma cruzi infection after heart transplantation. J Heart Lung Transplant. 2004;23:1345-8. 